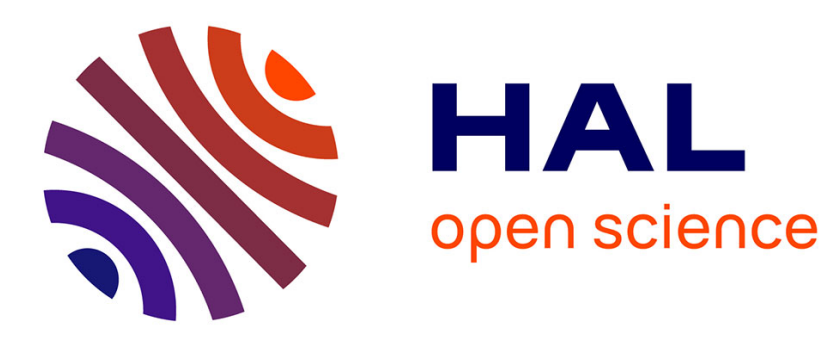

\title{
DCEA: a reference model for distributed ledger technologies
}

\author{
Badr Bellaj, Aafaf Ouaddah, Emmanuel Bertin, Noel Crespi, Abdellatif \\ Mezrioui
}

\section{- To cite this version:}

Badr Bellaj, Aafaf Ouaddah, Emmanuel Bertin, Noel Crespi, Abdellatif Mezrioui. DCEA: a reference model for distributed ledger technologies. ICBC 2021: IEEE International Conference on Blockchain and Cryptocurrency, May 2021, Sydney (online), Australia. pp.1-2, 10.1109/ICBC51069.2021.9461129 . hal-03168829

\section{HAL Id: hal-03168829 \\ https://hal.science/hal-03168829}

Submitted on 14 Mar 2021

HAL is a multi-disciplinary open access archive for the deposit and dissemination of scientific research documents, whether they are published or not. The documents may come from teaching and research institutions in France or abroad, or from public or private research centers.
L'archive ouverte pluridisciplinaire HAL, est destinée au dépôt et à la diffusion de documents scientifiques de niveau recherche, publiés ou non, émanant des établissements d'enseignement et de recherche français ou étrangers, des laboratoires publics ou privés. 


\title{
DCEA: A Reference model for Distributed Ledger Technologies
}

\author{
Badr Bellaj ${ }^{\$ *}$, Aafaf Ouaddah*, Emmanuel Bertin ${ }^{\S}$ (IEEE Senior), Noel Crespi ${ }^{\$}$ (IEEE Senior), Abdellatif Mezrioui ${ }^{*}$ \\ ${ }^{\$}$ Telecom SudParis, Paris, France, ${ }^{*}$ Institut National des Postes et Télécommunications, Rabat, Morocco, ${ }^{\S}$ Orange, France \\ Corresponding e-mail: bellaj.badr@mchain.uk
}

\begin{abstract}
Bitcoin's notable success at operating autonomously as a global peer-to-peer network opened the door to a new revolutionary class of distributed systems: blockchain. However, with the rising popularity of this new paradigm, the concept of blockchain shifted greatly to the point where many systems marketed as "blockchain" without sharing the key components introduced by Bitcoin. It has therefore become quite challenging for system designers or decision-makers to achieve a sufficient understanding of different types of existing blockchains or to design and adopt the correct blockchain solution. To overcome this problem, we propose DCEA a holistic reference model for conceptualizing and closely examining blockchains and globally distributed ledger technologies (DLT) using a layer-wise framework that envisions all these systems as constructed of four layers: the data, consensus, execution and application layers.
\end{abstract}

Index Terms-blockchain, DLT, reference model.

\section{INTRODUCTION}

Although there are multiple proposals for standardizing blockchain (ISO [1]-[2], IEEE [3], ITU [4] ), there is still no recognized standard for defining blockchain or DLT. The lack of a unified standard leads to growing use of ambiguous, imprecise, and inconsistent language and terminology across different projects - where the same term may be used to refer to different things - which could hinder the development of the DLT sector and its mass adoption.

Being aware of this, we introduce in this paper a reference model capturing a longitudinal and representative view of DLT systems. It proposes a systematic and holistic approach to conceptualize and examine DLTs in general as a functioning system with key layers at four levels of analysis.

\section{DCEA FRAMEWORK}

We propose DCEA, a framework that defines a layered heterogeneous stack for DLT systems. From a design perspective, our conceptual framework (DCEA) segregates DLT technologies into four essential and distinct layers: data, distributed consensus protocols, execution and application layers - each one playing a well-defined role in the DLT architecture. The framework consists of the DLTs components and their main properties (Table I], with logically related functions grouped together. This layering approach will help to provide a better understanding of DLTs, and serves as a baseline to build a comparative analogy between different DLT variants.

978-0-7381-1420-0/21/\$31.00 @2021 IEEE
In the following, we introduce the four layers that form the DLT stack.

- Data Layer: Represents the data flowing through the distributed network and stored in the ledger. Data in this layer is represented by entries recorded partially or completely in the ledger, under consensus and shared amongst the network participants. These records may represent elements defined by the underlying protocols (such as tokens), or data received from external environments (such as IoT data).

- Consensus layer: Defines the global software-defined ruleset to ensure agreement among all participants, in a network, on a unified ledger.

- Execution layer: Represents the components responsible for enforcing and executing distributed programs (e.g. smart contracts). These programs or contracts codify a given logic (e.g. a business logic) as a set of instructions for manipulating the states recorded in the ledger.

- Application layer: Represents an abstraction layer that specifies a variety of protocols and APIs provided by the DLT system to enable the building of distributed applications commonly called DApps. This layer also represents a communication link between the external actors or applications and the DLT ledger.

\section{DATA LAYER}

In this section, we lay out the key components, and their characteristics, that construct the data layer as introduced in Table I]

a) Data structure: DLT's data organization in its macroscopic structure varies from one technology to another. Generally, we distinguish between two main models of data structures in the DLT space; the linear chain of blocks (Blockchain) and the chain-less models.

b) State management: A key distinguishing factor among various DLTs is how states are managed within the system (on-chain). Although DLTs serve as distributed ledgers for shared data, in the case of many DLTs, data is stored outside the transactional distributed ledger (off-chain) in an auxiliary database.

c) Data shareability: All nodes in a DLT network exchange transactions carrying shared data to reach consensus, but due to privacy reasons different visions of data shareability have been adopted. 
TABLE I

LAYERS AND COMPONENTS OF DCEA FRAMEWORK

\begin{tabular}{|c|c|c|c|c|c|c|c|c|c|}
\hline Application Layer & \multicolumn{3}{|c|}{ Integrability } & \multicolumn{3}{|c|}{ DLT orientation and purpose } & \multicolumn{3}{|c|}{ Wallet and identity management } \\
\hline Execution Layer & \multicolumn{2}{|c|}{$\begin{array}{l}\text { Execution } \\
\text { environment }\end{array}$} & \multicolumn{2}{|c|}{ Turing-completeness } & \multicolumn{2}{|c|}{ Determinism } & \multirow{2}{*}{$\begin{array}{l}\text { Openness } \\
\begin{array}{l}\text { Governance } \\
\text { model }\end{array}\end{array}$} & \multicolumn{2}{|c|}{ Interoperability } \\
\hline Consensus Layer & Safety & Liveness & Finality & $\begin{array}{l}\text { Network } \\
\text { model }\end{array}$ & $\begin{array}{l}\text { Failure } \\
\text { model }\end{array}$ & $\begin{array}{l}\text { Adversary } \\
\text { model }\end{array}$ & & $\begin{array}{l}\text { Transaction } \\
\text { ordering }\end{array}$ & $\begin{array}{l}\text { Conflict } \\
\text { resolution }\end{array}$ \\
\hline Data Layer & \multicolumn{2}{|c|}{ Data structure } & \multicolumn{2}{|c|}{ Data shareability } & \multicolumn{2}{|c|}{ Data immutability } & \multicolumn{2}{|l|}{ Data privacy } & States management \\
\hline
\end{tabular}

d) Data immutability / Atomicity: There is a common belief that records stored on a DLT (especially a blockchain) are immutable and unalterable. However, under some circumstances, nodes can hold inconsistent states, or that a confirmed transaction may be reversed.

e) Data privacy: Data privacy is securing data from public view. In a shared context like in DLTs, data can be private or not private. This is possible with cryptographic Zeroknowledge techniques, which enable verifying private data without revealing it in its clear form.

\section{CONSENSUS LAYER}

In this section, we present the properties and features we consider as part of the DCEA framework for studying and differentiating between the consensus protocols.

a) Safety: Safety represents in the context of DLT networks, the guarantee that the correct nodes will not validate conflicting outputs (or make conflicting decisions) at the same time (e.g. chain forks).

b) Liveness: A consensus protocol guarantees liveness if requests (transactions) from correct clients are eventually processed.

c) Finality: the finality property is the affirmation and the guarantee for a transaction to be considered by the system as final and irreversible.

d) Network models: In DCEA, we consider the messagepassing model in which nodes exchange messages over the network, under differing assumptions of network synchrony.

e) Failure Models: we consider two main failure models: Fail stop failure and Byzantine faults.

f) Adversary models: The adversary is able to learn the message exchange between neighbours and corrupt different parts of the network.

g) Governance Model: The governance model refers to the process of decision-making adopted by a DLT network to decide on the protocol rules and their upgrade.

h) Identity Model: DLT protocols manage nodes membership differently, but in general two opposite approaches are adopted: Permissionless and permissioned.

i) Transactions ordering: Whether for a linear or a nonlinear DLT (e.g. DAGs), the stored transaction should be ordered chronologically to avoid frauds and inconsistencies.

j) Conflict resolution model: In some DLT networks, conflicting temporary versions of the ledger (known as forks) can coexist for different reasons. To converge toward a canonical ledger or chain, networks and consensus mechanisms adopt different rules to resolve the conflict.

\section{EXECUTION LAYER}

In a DLT system, business logic, agreed to by counterparties, can be codified using a set of instructions and embedded into the ledger in a specific format. The ruleset execution is enforced by the distributed consensus mechanism, thereby an external actor cannot influence or corrupt the execution of the instruction set to get advantageous results. Generally, we distinguish between two main models for rules codification: Smart contracts and built-in scripts.

\section{APPLICATION LAYER}

In this section, we briefly introduce the components and properties our DCEA framework defines for the application layer.

a) Integrability: DLT systems try to offer a better user experience by providing necessary tools (APIs, frameworks, protocols.) to enable better integrability with existing technologies (e.g. Web, mobile).

b) DApp orientation and DLT's purpose : Decentralized applications (or DApps for short) are software applications whose server and client tiers are decentralized and operating autonomously, with no controlling entity.

c) Wallet and identity management: Wallets are entities responsible for all cryptographic operations related to the creation or storage of the user's keys or digital certificates as well as the management of transactions.

\section{CONCLUSION}

In this paper, we have proposed a comprehensive and referential framework to ease the understanding and the investigation of the different approaches adopted by different DLTs at the four layers: data structure, execution, consensus and application layers. The layer-wise approach adopted by DCEA is aligned with the DLT's modular architecture and will help to provide a better and modular understanding of DLTs to decision-makers, who could then make granular decisions at each layer to construct the best solution.

\section{REFERENCES}

[1] E. N. Dawson, A. Taylor, and Y. Chen, "ISO/TC 307 Blockchain and distributed ledger technologies." [Online]. Available: https://www.iso. org/committee/6266604.html

[2] ISO/TR, "ISO/TR 23455:2019 Blockchain and distributed ledger technologies - Overview of and interactions between smart contracts in blockchain and distributed ledger technology systems."

[3] I. S. Association, "IEEE blockchain standards." [Online]. Available: https://blockchain.ieee.org/standards

[4] ITU, "Focus Group on Application of Distributed Ledger Technology." [Online]. Available: https://www.itu.int/en/ITU-T/focusgroups/dlt/Pages/ default.aspx 